\title{
ON CONTIGUOUS POINT SPACES
}

\section{THEODORE HAILPERIN}

In this paper we shall briefly indicate the kind of geometry obtained by a modification of one of Hausdorff's axioms for topological space. The resulting space turns out to be the contiguous point space of R. L. Moore (Rice Institute Pamphlet, vol. 23 (1936)). However, unlike Moore, we shall define contiguity, and we shall define it in terms of point and neighborhood. It is in terms of these two primitive indefinables that Hausdorff states his axioms for topological space (Mengenlehre, p. 228):

Axiom 1. Every point $p$ has a neighborhood $U_{p}$. For every $p, p \varepsilon U_{p}$.

Axiom 2. For every two neighborhoods $U_{p}$ and $V_{p}$ of the same point, there is a third $W_{p} \subset U_{p} \cdot V_{p}$.

Axıом 3. Every point $q \varepsilon U_{p}$ has a neighborhood $U_{q} \subset U_{p}$.

Axiom 4. For every $p$ and $q, p \neq q$ implies that there exist neighborhoods $U_{p}$ and $U_{q}$ such that $U_{p} \cdot U_{q}=0$.

A contiguous point space will be defined by the Axioms 1-3 to. gether with the following new axiom:

Axıом $4{ }^{\prime}$. There exist points, for example, $p$ and $q$, such that $p \neq q$ and such that for every $U_{p}$ and $U_{q}$ the common part $U_{p} \cdot U_{q}$ contains both $p$ and $q$.

This axiom is obtained by negating 4 and substituting the condition $U_{p} \cdot U_{q} \supset(p, q)$ for the weaker condition $U_{p} \cdot U_{g} \neq 0$. The property given by $4^{\prime}$ approximates our ordinary idea of contiguity; we set this down as a formal definition.

Definition. The point $p$ is said to be contiguous to the point $q$ if (1) $p \neq q$, and (2) any neighborhood of the one point contains the other.

First, it may be pointed out, no space containing contiguous points can be a topological space. This is obvious from the method of deriving $4^{\prime}$. In topological space a set must have at least a denumerable infinity of points in order for it to have a limit point. This is not true for contiguous points since, if $p$ and $q$ are contiguous, the point $p$ is a limit point of the set $(q)$, which is a set containing only one point.

THEOREM 1. No point is contiguous to itself. 
THeorem 2. If a point $p$ is contiguous to $q$, then $q$ is contiguous to $p$.

Theorem 1 results immediately from the first part of the definition of "contiguous to" while Theorem 2 is a consequence of the symmetry of both parts.

Definitron. The point $p$ is said to be contiguous to the set $M$ if $p$ is contiguous to some point which is an element of $M$.

Theorem 3. If $p$ is contiguous to $M$, then $p$ is a limit point of $M$.

Proof. Let $q \varepsilon M$ be a point which is contiguous to $p$. Then every neighborhood of $p$ contains $q$, which is an element of $M$. Hence $p$ is a limit point of $M$.

THEOREM 4. If $M$ is a closed point set and every point of the set $H$ is contiguous to $M$, then no point of $1-M$ is a limit point of $H$.

Proof. By Theorem 3, every point of $H$ is a limit point of $M$. Since $M$ is closed, $H$ is contained in $M$ and consequently can have no limit points in $1-M$.

Theorems 1, 2, and 4 were taken as axioms by Moore. This was necessary since contiguity was an undefined concept in his system. By defining "contiguous to" in terms of "point" and "neighborhood" we were able to derive these three propositions from one axiom, namely, $4^{\prime}$. In addition we are saved the trouble of redefining a number of concepts such as "connectivity" and "boundary point"; for example, the idea of contiguity was used by Moore chiefly in relation with connectivity. Ordinarily, the sets $A$ and $B$ are said to be mutually separated if they satisfy the following conditions:

(1) They are mutually exclusive.

(2) Neither of them contains a limit point of the other.

To these Moore adds the further condition:

(3) No point of $A$ is contiguous to any point of $B$.

Thus the notion of connectedness, which is defined in terms of this definition acquires a new significance in spaces containing contiguous points. A set is said to be connected if and only if it is not the sum of two mutually separated sets.

The additional condition (3) which Moore had to assume now becomes unnecessary since it results from the other two in conjunction with Theorem 3. This may be stated as a theorem: 
TheOREM 5. If $M$ and $N$ are connected sets and there exists a point $p \varepsilon M$ which is contiguous to $N$, then $M+N$ is connected.

THEOREM 6. If $p \varepsilon 1-M$ and $p$ is contiguous to $M$, then $p$ is a boundary point of $M$.

This results immediately from Theorem 3 . Here again we have derived a property which Moore had to assume (by enlarging the definition of "boundary point," loc. cit., p. 7).

THEOREM 7. If $p$ is contiguous to $q$, the set $(p, q)$ is connected.

TheOREM 8. If $p$ is contiguous to $q$ and there exists a neighborhood containing $p$ and $q$ but no other contiguous points besides $p$ and $q$, then the set $(p, q)$, consisting solely of $p$ and $q$, is closed, connected, and compact; that is, is a simple continuous arc from $p$ to $q$.

PRoof. The set is closed since the only limit points which $(p, q)$ may have must be contiguous to $p$ or $q$ and this is ruled out by hypothesis. The set is connected by Theorem 8 , and, finally, is obviously compact since it contains no infinite subset.

These theorems suffice to indicate the type of geometry which contiguity involves. For some important applications the reader is recommended to the aforementioned work of R. L. Moore.*

UnIVERSITY OF Michigan

* At an advanced stage in his paper, Moore introduces a restrictive axiom (loc. cit. p. 39):

Axıм D. There do not exist 3 distinct points such that each of them is contiguous to each of the others.

This axiom can only be proved here by assuming that our points are linearly ordered. 